i) 当 $3+\bar{D}$ 时, $(3 \bar{D}, 3 \bar{x}, 9 \bar{y})$ 为 $\left(2^{\prime}\right)$ 之解;

[2] 柯 召、孙 琦, 中国科学, 1981, (12): 1453-1457.

ii) 当 $3+\bar{D}$ 时, $(3 \bar{D}, 3 \bar{x}, 3 \bar{y})$ 为 $\left(2^{\prime}\right)$ 之解.

\section{参考文献}

[1] Ljunggen, W. J., Sate uber Unbestimmte Gleichungen, Skey Norskevid Aked, Oslo 1, 1942, 9: 53.
李复中

(东北师范大学数学系, 长春 130024 )

\title{
低维有限点集偏差的精确计算公式 (III)
}

本通信是文献 [1] 和[2] 的继续. 设 $d \geqslant 2$, $S_{d}=\left\{u_{k}(1 \leqslant k \leqslant n)\right\}$ 是 $d$ 维单位立方体 $G_{d}=[0$, $1)^{d}$ 中的有限点集, $\boldsymbol{u}_{k}=\left(u_{1, k}, u_{2, k}, \cdots, u_{d, k}\right)$ 满 足 $u_{1,1} \leqslant u_{1,2} \leqslant \cdots \leqslant u_{1, n}$. 令 $u_{0}=\left(u_{1,0}, u_{2.0}, \cdots\right.$, $\left.u_{\alpha, 0}\right)=(0,0, \cdots, 0), u_{n+1}=\left(u_{1, n+1}, u_{2, n+1}, \cdots, u_{d, n+1}\right)$ $=(1,1, \cdots, 1)$. 对于每个 $l_{1}\left(l_{1}=0,1, \cdots, n\right)$ 按 递增顺序排列 $u_{2, j}\left(j=0,1, \cdots, l_{1}, n+1\right)$, 并将它 们记作

$$
0=\bar{u}_{2,1_{1}, 0} \leqslant \bar{u}_{2,1_{1}, 1} \leqslant \cdots \leqslant \bar{u}_{2,1_{1}, l_{1}}<\bar{u}_{2, l_{1}, l_{1}+1}=1,
$$

而相应的 $x_{\tau}$ 莝标 $u_{\tau, j}$ 记作 $u_{\tau, l_{1}, \sigma_{1}(j)}\left(j=0, \cdots, l_{1}\right.$, $\left.l_{1}+1\right)$, 其中 $\tau=3, \cdots, d, \sigma_{1}$ 是由上述排列决定 的 $\left\{0, \cdots, l_{1}, l_{1}+1\right\}$ 的一个置换. 对于每个固定 的 $l_{1}\left(0 \leqslant l_{1} \leqslant n\right)$, 对任何 $l_{2}\left(l_{2}=0,1, \cdots, l_{1}\right)$ 按递 增顺序排列 $u_{3, l_{1}, \sigma_{1}(0)}\left(j=0, \cdots, l_{2}, l_{1}+1\right)$, 并将它 们记作

$0=\bar{u}_{3, l_{1}, l_{2}, 0} \leqslant \bar{u}_{3, l_{1}, l_{2}, 1} \leqslant \cdots \leqslant \bar{u}_{3, l_{1}, l_{2}, l_{2}}<\dot{\bar{u}}_{3, l_{1}, l_{2}, l_{2}+1}=1$, 而相应的 $x_{\tau}-$ 坐标 $u_{\tau, j}$ 记作 $u_{\left.\tau, l_{1}, l_{2}, \sigma_{2}()\right)}\left(j=0, \cdots, l_{2}\right.$, $\left.l_{2}+1\right)$, 其中 $\tau=4, \cdots, d, \sigma_{2}$ 是由上述排列决 定的 $\left\{0, \cdots, l_{2}, l_{2}+1\right\}$ 的一个置换. 上述过 程可逐次进行. 一般地, 设 $1<v<d-1$, 且对任 何 $v$ - 数组 $\left(l_{1}, l_{2}, \cdots, l_{v}\right)$, $n \geqslant l_{1} \geqslant l_{2} \geqslant \cdots \geqslant l_{v} \geqslant 0$, 我们已经逐次按递增顺 序排列了坐标 $u_{2, j}\left(j=0, \cdots, l_{1}, n+1\right), u_{3, l_{1}, \sigma_{1}(j)}(j=0$, $\left.\cdots, l_{2}, l_{1}+1\right), \cdots$, 以及 $u_{v+1, l_{1}, \cdots, l_{v+1}, \sigma_{v-1}(j)}\left(j=0, \cdots, l_{v}\right.$, $\left.l_{v-1}+1\right)$, 其中 $\sigma_{i}$ 是 $\left\{0, \cdots, l_{t}, l_{t}+1\right\}$ 的一个置换 $(t=1,2, \cdots, v-1)$, 并且分别把它们记作

$$
\begin{aligned}
0= & \bar{u}_{r, l_{1} \cdots, l_{r-1} .0} \leqslant \bar{u}_{r, l_{1} \cdots, l_{r-1}, 1} \leqslant \cdots \leqslant \bar{u}_{r, l_{1}, \cdots, l_{r-1}, l_{r}-1} \\
& <\bar{u}_{r, l_{1} \cdots, l_{r}-1} l_{r-1}+1=1(r=2,3, \cdots, v+1),
\end{aligned}
$$

那么对任何 $l_{v+1}\left(l_{v+1}=0, \cdots, l_{v}\right)$, 我们按递增顺
序排列坐标 $\dot{u}_{v+2, I_{1}, \cdots, I_{v}, \sigma_{v}(j)}\left(j=0, \cdots, l_{v+1}, l_{v}+1\right)$, 其中 $\sigma_{v}$ 是 $\left\{0, \cdots, l_{v}, l_{v}+1\right\}$ 的一个置换, 并且将 它们写作

$$
\begin{gathered}
0=\bar{u}_{v+2, I_{1}, \cdots, l_{v+1}, 0} \leqslant \bar{u}_{v+2, I_{1}, \cdots, l_{v+1,1}} \leqslant \cdots \\
\leqslant \bar{u}_{v+2, l_{1}, \cdots, l_{v+1}, l_{v+1}}<\bar{u}_{v+2, I_{1}, \cdots, l_{v+1}, l_{v+1}+1}=1 .
\end{gathered}
$$

于是对每个 $(d-1)$ 一数组 $l=\left(l_{1}, \cdots, l_{d-1}\right), n \geqslant l_{1}$ $l_{2} \geqslant \cdots \geqslant l_{d-1} \geqslant 0$, 我们可逐次得到 $d-1$ 个数 集 $\bigcup_{l, r}=\left\{\bar{u}_{r+1 . l_{1} \ldots l_{n} j}\left(j=0, \cdots, l_{r}, l_{r}+1\right)\right\}, r=1, \cdots$, $d-1$, 适合 .

$$
\begin{gathered}
0=\bar{u}_{r+1, l_{1}, \cdots, l_{r} 0} \leqslant \bar{u}_{r+1, l_{1}, \cdots, l_{r}, 1} \leqslant \cdots \leqslant \bar{u}_{r+1, l_{1}, \cdots, I_{n} l_{r}} \\
<\bar{u}_{r+1 . l_{1}, \cdots, l_{r}, l_{r+1}}=1 .
\end{gathered}
$$

定理 设 $S_{d}$ 及数集 $U_{t . r}$ 定义如上, 则 $S_{d}$ 的偏差由下式给出

$$
\begin{gathered}
D_{n}\left(S_{d}\right)=\max _{0 \leqslant k_{1} \leqslant n} \max _{0 \leqslant k_{2} \leqslant k_{1}} \cdots \max _{0 \leqslant k_{d} \leqslant k_{d-1}} \max \\
\left(\frac{k_{d}}{n}-u_{1, k_{1}} \bar{u}_{2, k_{1}, k_{2}} \cdots \bar{u}_{d, k_{1}, \cdots, k_{d}}, u_{1, k_{1}} \bar{u}_{2, k_{1}, k_{2}+1} \cdots\right. \\
\left.\bar{u}_{d, k_{1}, \cdots, k_{d-1} \cdot k_{d-1} \cdot k_{d}+1}-\frac{k_{d}}{n}\right) .
\end{gathered}
$$

特别, 若 $\boldsymbol{u}_{1}=(0, \cdots, 0)$ 则上式右边取 $\max$ 的 范围可换为 $1 \leqslant k_{1} \leqslant n, 1 \leqslant k_{2} \leqslant k_{1}, \cdots, 1 \leqslant k_{d}$ $\leqslant k_{d-1}$.

例 5 维点集 $S_{5}=\left\{\left(\frac{k}{31},\left\{\frac{2}{31} k\right\}\right.\right.$, $\left.\left.\left\{\frac{4}{31} k\right\},\left\{\frac{8}{31} k\right\},\left\{\frac{16}{31} k\right\}\right)(1 \leqslant k \leqslant 31)\right\}$ 有 偏差 $D_{31}=0.2797$. 
参考文献

[1] Bundschuh, P., 朱尧辰, 科学通报, 1993, 38(7): 669 .

[2] Bundschuh, P., Zhu, Y., Abh. Math. Univ.
Hamburg, 1993, 63: 115-133.

\section{朱尧辰}

(中国科学院应用数学研究所, 北京 100080)

\section{寻找油气富集带的遥感找矿新方法 卫星热红外探测技术 *}

油气富集带不断向地表进行放气作用, 因此经常逸出 $\mathrm{CO}_{2}, \mathrm{CH}_{4}$ 等气体. 这各种地 球化学成分的异常, 以前人们是从地表的调 查直接获得的. 例如: 1953 年美国 Fleizcher 和Turner ${ }^{[1]}$ 利用土壤中碳同位素 ${ }^{13} \mathrm{C} /{ }^{14} \mathrm{C}$ 变 化,在美国纽约州西北部找出油气藏. 而前 苏联 C.M. AmocoB等在白俄罗斯波列西耶 油气区利用人工震源来测量含油气盆地内 $\mathrm{CH}_{4}, \mathrm{CO}_{2}$ 溢出量变化来圈出油气藏范围取 得满意效果 ${ }^{[2]} .1992$ 年刘燕君等在卫星遥感 红外图像上也发现油气显示 ${ }^{[3,4]}$.

天然地震如同人工震源, 只是震动时间 前者是无法预知的. 由于现代技术的进展, 从 我们从事地震临震预报的长期工作中发现, 油气盆地的这种地表气体地球化学异常完全 可以用现代高技术的卫星遥感技术来检测.

下面用两个地质实例来说明检测结果.

(1) 松辽盆地: 1990 年 5 月 11 日发生了 我国东北深震区日本海 6.0 级地震, 在其西 侧 $1000 \mathrm{~km}$ 的内蒙古开鲁、鲁北一带, 震前 4 天出现了一个近北西向、长轴近 $300 \mathrm{~km}$; 短 轴为北东向、长约 $200 \mathrm{~km}$ 的椭圆形即卫星 热红外增温异常区, 此异常区位于开鲁抝陷 ${ }^{[s]}$. 1991 年石油部门在开鲁地区钻探中打出具有 工业价值油气来, 说明利用卫星的热红外增温 异常来寻找富聚油气的可行性及科学性.

(2) 塔里木盆地: 1991 年 6 月 17 日在新 疆塔里木盆地南缘民丰发生了 5.3 级地震, 震前数日在塔里木盆地于田、民丰北侧出现了
南北 宽 $200 \mathrm{~km}$, 东西长约 $300 \mathrm{~km}$ 的椭圆形 增温异常区 (图 1), 具体位置在 N37-39 , $\mathrm{E} 80.5-83.5^{\circ}$. 塔里木盆地可划分为七大构 造单元: (1) 西南拗陷, (2) 巴楚隆起, (3) 东南 断块隆起, (4) 塘古巴斯断陷, (5) 塔东断陷, (6) 塔北隆起, (7) 库车拗陷 ${ }^{[6}$.

古生代、中生代及新生代时期巨厚陆屑 及多期海相层堆积, 早期堆积为生油条件, 后期为储集层和储油构造. 因此西南拗陷东 端、民丰抝陷中的于田一民丰增温异常区为 一油气富集带.

卫星热红外探测技术是一种既经济、又

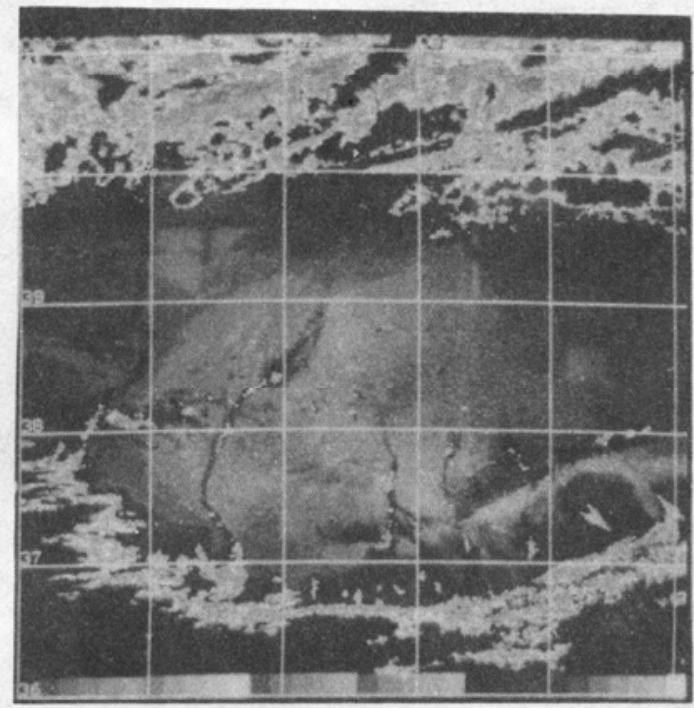

图 1 1991 年 6 月 17 日 05 h $57 \mathrm{~min}$ 气象卫星 热红外黑白图象

灰阶从左端起始温度为 $-2^{\circ} \mathrm{C}$, 向左每小方格增温 $1^{\circ} \mathrm{C}$ 